\title{
La prestación económica de asistencia personal en el territorio histórico de Gipuzkoa
}

\section{Zorione Benedicto Astorkia}

Departamento de Política Social, Diputación Foral de Gipuzkoa

<zbenedicto@gipuzkoa.net>

Artikulu honek aztertzen du Gipuzkoako mendekotasun-egoeran dauden pertsonei laguntzeko diru-prestazioaren kudeaketa-modeloa, berau abiarazi zela sei urte betetzear direnean. Modeloaren eta prestazioaren garrantziaren funtzionamendua deskribatu ondoren, DAFO metodoa eta arreta pertsonaleko zerbitzuak eskaintzen dituzten enpresetako elkarrizketak erabiltzen dira bertan, hobekuntza-proposamen batzuk ezartzeko.

\section{HITZ GAKOAK:}

Mendekotasuna, asistentzia pertsonala, asistentzia pertsonalerako prestazio ekonomikoa, kudeaketaren eredua, DAFO analisia, zerbitzuen plangintza.
Este artículo examina el modelo de gestión de la prestación económica para la asistencia a personas en situación de dependencia en Gipuzkoa, cuando se van a cumplir seis años de su puesta en marcha. Tras describir el funcionamiento del modelo y el alcance de la prestación, se utiliza el método DAFO y las entrevistas a empresas que ofrecen servicios de atención personal, para establecer algunas propuestas de mejora.

\section{PALABRAS ClaVe:}

Dependencia, asistencia personal, prestación económica de asistencia personal, modelo de gestión, análisis DAFO, planificación de servicios. 


\section{Introducción}

El desarrollo de la Ley 39/2006 para la Promoción de la Autonomía y Atención a las Personas en situación de dependencia y, en concreto, la prestación económica de asistencia personal, ha favorecido que personas dependientes puedan contratar a asistentes personales para su atención.

En el territorio histórico de Gipuzkoa, dicha prestación se ha desarrollado ampliamente y sus solicitudes suponen el $15 \%$ de las de todas las prestaciones económicas de dependencia en vigor. En el resto de las comunidades autónomas del Estado, en cambio, el desarrollo de esta ayuda ha sido escaso y sus solicitudes representan un porcentaje muy pequeño. Tal es así que en estos momentos más del $85 \%$ de las prestaciones económicas de asistencia personal de todo el Estado corresponden a Gipuzkoa (Imserso, 2013). El motivo fundamental es que la atención no se da únicamente para el apoyo en los ámbitos laboral y educativo, sino que se hace una lectura amplia de la cobertura y se atienden las necesidades básicas de la persona en su entorno, a través de la contratación de personas o empresas de servicios.

El modelo de gestión que hasta el momento se ha utilizado ha venido a cubrir una demanda emergente muy importante, a la que se ha dado prioridad. La gestión de la prestación es competencia exclusiva del Departamento de Política Social de la Diputación Foral de Gipuzkoa, pero depende, en gran medida, de factores como la coordinación con las empresas de servicios de atención a la dependencia, la población potencialmente contratable para estas tareas de cuidado o los requisitos para darse de alta como trabajador/a en la Seguridad Social.

Este artículo se basa en un estudio realizado en mayo de 2013 sobre la gestión y mejora de la prestación económica de asistencia personal en Gipuzkoa ${ }^{1}$, como recurso para apoyar la permanencia en su entorno de las personas en situación de dependencia o con diversidad funcional. Dicho estudio se presentó como trabajo de fin de curso para la obtención del título de grado en Trabajo Social de la Universidad de Deusto (Donostia).

El artículo comienza describiendo el modelo utilizado por el Departamento de Política Social de la Diputación de Gipuzkoa para gestionar la prestación económica de asistencia personal. En segundo lugar, se ofrecen algunas cifras sobre el alcance de la prestación, las personas beneficiarias y los/las asistentes personales contratados. Una vez presentado el objeto de estudio, se ofrece el análisis DAFO del modelo de gestión, se resumen los principales datos aportados por las entrevistas y se exponen algunas conclusiones generales. Para terminar, se proponen una serie de mejoras en el modelo de gestión previamente examinado.

${ }^{1}$ Los datos estadísticos de dicho trabajo, que corresponden a 2012, se compararán en este artículo con las cifras más recientes, obtenidas en noviembre de 2013

\section{Modelo de gestión de la prestación económica de asistencia personal en el Departamento de Política Social}

En este apartado, se presentan los agentes que intervienen en el modelo de gestión de la prestación económica de asistencia personal en Gipuzkoa: el Departamento de Política Social de la Diputación, la Sección de Prestaciones Económicas de Dependencia, la ciudadanía y los servicios sociales de base.

\subsection{Departamento de Política Social}

El Departamento de Política Social de Diputación Foral de Gipuzkoa tiene competencias en materia de servicios sociales (atención secundaria/especializada), según la Ley 12/2008, de Servicios Sociales del País Vasco, así como en la gestión de las prestaciones económicas para la asistencia a personas en su entorno natural. Respecto a la Sección de Prestaciones de Dependencia, sus funciones son:

- Gestionar el presupuesto de la sección².

- Emitir las resoluciones de concesión, denegación, modificación de cuantías y cambios de asistente personal, así como comunicárselas al ciudadano a propuesta de la Sección de Prestaciones de Dependencia.

\subsection{Sección de Prestaciones Económicas de Dependencia}

La Sección de Prestaciones Económicas de Dependencia tiene encomendada la gestión de las prestaciones económicas de dependencia, la pensión no contributiva y el complemento de vivienda, el subsidio de movilidad y los gastos transporte y asistencia sanitaria de la Ley de Integración Social del Minusválido, y el Fondo de Bienestar Social. En 2012, atendió a $\mathbf{1 5 . 0 0 0}$ personas y tuvo un gasto de 63.571.972,34 euros.

Las principales funciones de la Sección son:

- Recepción de solicitudes a través de una aplicación informática en línea.

- Gestión: revisión de documentación, propuesta de concesión económica o denegación a la vista de la petición, actualizaciones/modificaciones de las solicitudes. Actualmente no se hace un trabajo de gestión de caso, en la medida que sólo se tienen en cuenta gastos y cuantías concedidas.

- Asesoramiento al ciudadano y a los trabajadores de los servicios sociales de base: información sobre la prestación, trámites necesarios, derivación al Ayuntamiento, justificación de gastos.

- Revisión anual de los gastos justificados.

${ }^{2}$ En concreto, el presupuesto de 2012 para la prestación económica de asistencia personal ascendía a 8.950.241,37 €. 
- Revisión de oficio del cumplimiento de los requisitos: referencias a las altas y bajas de trabajadores en la Seguridad Social, y al empadronamiento de los solicitantes en el territorio de Gipuzkoa.

\subsection{Ciudadanía}

La prestación económica de asistencia personal está contemplada en la Ley 39/2006 como un derecho para toda persona que cumpla los siguientes requisitos:

- Estar en situación de dependencia o tener diversidad funcional, de acuerdo el baremo de valoración de dependencia (Real Decreto 504/2007). Actualmente, en Gipuzkoa tienen derecho a servicios o prestaciones las personas en situación de dependencia con, al menos, un grado I y 40 puntos ${ }^{3}$.

- Necesitar asistencia personal para llevar una vida más autónoma.

- Tener al menos 3 años de edad.

- Contratar a una persona:

a. bien directamente, por medio de un contrato legal y con alta en la Seguridad Social (régimen de empleadas de hogar o autónomos);

b. bien a través de una empresa de servicios de asistencia personal. En este caso, el contrato es firmado entre la empresa y la persona en situación de dependencia. Los trabajadores dependen de la empresa, que se ocupa del contrato y el alta en la Seguridad Social. El contrato entre la empresa y la persona en situación de dependencia fija el horario, las tareas y el sueldo, entre otras cuestiones.

\subsection{Servicios sociales de base (ayuntamientos)}

Los servicios sociales de base tienen competencias en materia de servicios sociales de atención primaria, primer eslabón de la cadena del Sistema Vasco de Servicios Sociales. Sus funciones generales incluyen la información, el asesoramiento, la orientación, el seguimiento y la supervisión. En lo que se refiere específicamente a la prestación económica de asistencia personal, los servicios sociales de base se ocupan de:

- La tramitación de solicitudes.

- El seguimiento de las prestaciones económicas concedidas, con el fin de dar respuesta a la demanda del ciudadano, mejorar la calidad de vida de la persona en situación de dependencia, y colaborar en la gestión de la eficacia y eficiencia de la Administración.

3 Según el calendario de implantación progresiva de la Ley 39/2006, actualmente sólo tienen derecho a las prestaciones las personas valoradas con los grados II y III. Las valoradas con el grado I (de 25 a 49 puntos) se incorporará en julio de 2015. La Diputación Foral de Gipuzkoa aprobó, a través de la modificación 2/2013 del Decreto Foral $25 / 2009$, la cobertura de prestaciones a personas valoradas como dependientes con grado I y un mínimo de 40 puntos.
- La derivación de las solicitudes a la Sección de Prestaciones Económicas de Dependencia de la Diputación Foral.

\section{La prestación económica de asistencia personal en Gipuzkoa: algunas cifras}

La Tabla 1 sintetiza los datos más relevantes sobre las solicitudes de la prestación económica de asistencia personal realizadas en Gipuzkoa en los dos últimos años.

A 17 de noviembre de 2013, las solicitudes de prestación económica de asistencia personal realizadas en Gipuzkoa alcanzan las 1.832, de las cuales 923 corresponden a nuevas altas tramitadas durante ese año (el 50,5\% de las solicitudes actuales de alta). Otras 310 solicitudes de alta han pasado de tener una prestación para cuidados en el entorno familiar a una de asistencia personal, lo que supone el $33,58 \%$ del total de solicitudes de alta nuevas.

Las solicitudes tramitadas hasta 2013 debían tener un grado III de dependencia. El Real Decreto Ley 20/2012, de 13 de julio, de Medidas para garantizar la estabilidad presupuestaria y de fomento de la competitividad, establece varios cambios que afectan directamente a la Ley 39/2006, de 14 de diciembre, de Promoción de la Autonomía Personal y Atención a las personas en situación de dependencia. Estos cambios fueron asumidos por la Diputación Foral de Gipuzkoa mediante el Decreto Foral 2/2013, de 22 de enero, de modificación del Decreto Foral 25/2009, de 21 de julio, por el que se permite que cualquier persona con un grado de dependencia con puntuación superior a 40 puntos pueda solicitar una prestación económica de asistencia personal. Esta norma toma en cuenta, con carácter retroactivo, los contratos con alta en la Seguridad Social realizados desde agosto de 2012.

Del total de prestaciones de alta registradas hasta el 17 de noviembre de 2013 (1.832 solicitudes), el 6,8\% corresponden a personas con un grado I de dependencia; el 26,0\%, a personas con un grado II; y el $67,2 \%$, a personas con un grado III.

De la comparación entre los datos estadísticos de 2012 y 2013 , se pueden extraer las siguientes conclusiones:

- El perfil de la persona solicitante en 2012 era el de una mujer de más de 65 años en situación de gran dependencia (grado III), atendida por otra mujer extranjera, contratada directamente y con alta en el régimen de empleadas de hogar. Este perfil no ha variado casi nada en 2013 , salvo por el hecho de que, con el cambio introducido por el Decreto Foral 25/2009, ahora el $33 \%$ de las solicitudes de alta corresponden a personas con dependencia de grados I y II. 
Tabla 1. Principales cifras relativas a las solicitudes de prestaciones económicas de asistencia personal. Gipuzkoa, 2012-2013

\begin{tabular}{|c|c|c|c|c|c|}
\hline & & \multicolumn{2}{|c|}{ 2012* } & \multicolumn{2}{|c|}{$2013^{\star \star}$} \\
\hline & & Abs. & $\%$ & Abs. & $\%$ \\
\hline \multicolumn{6}{|c|}{ Personas beneficiarias } \\
\hline \multirow{2}{*}{ Sexo } & Mujer & 867 & 72,9 & 1.318 & 72,0 \\
\hline & Hombre & 323 & 27,1 & 514 & 28,0 \\
\hline \multirow{4}{*}{ Edad } & $<18$ años & 18 & 1,5 & 19 & 1,1 \\
\hline & 19-64 años & 45 & 3,7 & 100 & 5,4 \\
\hline & 65-79 años & 171 & 14,4 & 355 & 19,4 \\
\hline & $>79$ años & 956 & 80,3 & 1.358 & 74,1 \\
\hline \multicolumn{6}{|l|}{ Contratación } \\
\hline \multicolumn{2}{|c|}{ № de empresas contratadas } & 22 & - & 30 & - \\
\hline \multicolumn{2}{|c|}{ Personas que contratan con empresas } & 113 & 9,5 & 175 & 9,5 \\
\hline \multicolumn{2}{|c|}{$\begin{array}{l}\text { Personas que contratan directamente un/a } \\
\text { asistente personal }\end{array}$} & 1.077 & 90,5 & 1.657 & 90,5 \\
\hline \multicolumn{6}{|c|}{ Asistentes personales } \\
\hline \multirow{2}{*}{ Sexo } & Mujer & 1.034 & 96,0 & 1.567 & 94,5 \\
\hline & Hombre & 43 & 4,0 & 90 & 5,5 \\
\hline \multirow{2}{*}{ Nacionalidad } & Española & 314 & 29,2 & 521 & 31,5 \\
\hline & Extranjera & 763 & 70,9 & 1.136 & 68,6 \\
\hline
\end{tabular}

* Datos a 31 de diciembre de 2012. ** Datos a 17 de noviembre de 2013.

Fuente: Elaboración propia a partir de datos facilitados por la Sección de Prestaciones de Dependencia del Departamento de Política Social de la Diputación Foral de Gipuzkoa.

Tabla 2. Clasificación de las solicitudes de prestación económica de asistencia personal, según la fecha de solicitud y el grado de dependencia de la persona solicitante. Gipuzkoa, 2013

\begin{tabular}{l|c|c|c|c}
\hline \multirow{2}{*}{} & Nuevas & Anteriores a 2013* & \multicolumn{2}{c}{ Total } \\
\cline { 2 - 5 } & Abs. & Abs. & Abs. & 13,4 \\
\hline Grado I & 123 & 1 & 124 & 51,7 \\
\hline Grado II & 468 & 9 & 477 & 34,9 \\
\hline Grado III & 322 & 0 & 322 & 100,0 \\
\hline Total & 913 & 10 & 923 & \\
\hline
\end{tabular}

* Reactivación de bajas.

Fuente: Elaboración propia a partir de datos facilitados por la Sección de Prestaciones de Dependencia del Departamento de Política Social de la Diputación Foral de Gipuzkoa.

- Hay un ligero aumento en la proporción de personas que trabajan como asistentes personales autóctonos (31,44\% en 2013 y 29,15\% en 2012), lo mismo que en la proporción de asistentes personales varones (5,43\% en 2013 y $4 \%$ en 2012).

- Un $9,5 \%$ de las personas beneficiarias contratan una empresa de servicios en vez de contratar directamente a un/a asistente personal. Este porcentaje no ha variado de 2012 a 2013, pues el aumento registrado entre quienes realizan este tipo de contratación ha sido proporcional al del número de prestaciones económicas concedidas.

- El 33,5\% de las nuevas solicitudes de prestaciones de asistencia personal provienen de una prestación económica de cuidados en el entorno. Este trasvase ha hecho disminuir el número de prestaciones económicas de cuidado en el entorno que están de alta: a 31 de enero de 2012 había unas 10.200 solicitudes de alta, y en noviembre del año siguiente, 9.800 .

El Gráfico 1 muestra la evolución del gasto en prestaciones económicas de asistencia personal durante los últimos cinco años.

\section{Diagnóstico del actual modelo de gestión de la prestación económica de asistencia personal en Gipuzkoa}

Para realizar el diagnóstico del modelo actual, se ha utilizado una metodología que se puede dividir en cuatro fases:

- Elección de indicadores para realizar el diagnóstico, extraídos de la literatura sobre gestión de servicios sociales.

- Elaboración de un análisis DAFO del modelo de gestión basado en dichos indicadores.

- Entrevista a diez empresas proveedoras de servicios de asistencia personal para recabar datos sobre su funcionamiento y plantillas.

- Extracción de conclusiones y planteamiento de propuestas de mejora.

Los indicadores seleccionados se exponen en el Cuadro 1. 
*Datos a 31 de diciembre, salvo los de 2013, que son a 1 de noviembre.

Fuente: Elaboración propia a partir de datos facilitados por la Sección de Prestaciones de Dependencia del Departamento de Política Social de la Diputación Foral de Gipuzkoa.

Cuadro 1. Indicadores empleados para realizar el diagnóstico

Referidos al Departamento de Política Social y a la Sección de Prestaciones Económicas de Dependencia tudes recibidas y a las cuantías aprobadas de abono.

- Regulación reglamentaria de las empresas proveedoras de servicios de atención domiciliaria.

- Cumplimiento de los principios de la Ley

estatal 39/2006 y la Ley vasca 12/2008.

- Coordinación entre la Sección y los servicios

sociales de base municipales para la trami-

tación de las solicitudes.

- Impulso del actual Gobierno foral al desarro-

llo de la prestación.

- Cobertura de la prestación.
Referidos a las personas en situación de dependencia
$30 \%$

$25 \%$

$20 \%$

$15 \%$

$10 \%$

$5 \%$

$0 \%$

Fuente: Elaboración propia.

\section{Diagnóstico DAFO}

Para aplicar los indicadores antes señalados y conocer mejor la situación actual en el modelo de gestión de la prestación de asistencia personal, se ha elegido el análisis DAFO, cuyo objetivo es determinar:

- Debilidades: necesidades actuales que implican aspectos negativos que deberían modificarse.

- Amenazas: riesgos potenciales que deberían prevenirse.

- Fortalezas: aspectos positivos que deberían mantenerse o reforzarse.

- Oportunidades: capacidades y recursos potenciales que deberían aprovecharse.

\subsection{Debilidades}

5.1.1. Ausencia de planes de atención, proyecto de vida o plan individualizado de las personas en situación de dependencia que perciben prestación económica de asistencia personal

A la hora de realizar una solicitud de prestación económica de asistencia personal, entre la documentación que se debe presentar no se incluye ningún plan de atención de la persona en situación de dependencia. Es suficiente el contrato de trabajo, en el que no se especifica el nivel de atención que se presta, ni se diferencia si son tareas domésticas o de atención personal. Sin embargo, sería conveniente que la persona en situación de dependencia o su familia reflejara en 
un documento cuestiones como las necesidades que se requiere cubrir, las tareas de apoyo que se precisan y el horario del o de la asistente personal.

Para solicitar la ayuda económica del Programa de Vida Independiente, que se gestiona en el Departamento de Política Social, se realiza un proyecto de vida independiente que debe constar de los siguientes apartados (Decreto Foral 87/2008):

- Descripción de la situación de convivencia.

- Actividades ocupacionales o laborales que realiza la persona solicitante.

- Necesidades de atención: cuidados personales y tareas domésticas para las que precisa asistencia.

- Estimación del número de horas de atención que requiere para cubrir sus necesidades.

- Organización de los cuidados.

A la hora de demandar una prestación económica de asistencia personal sería necesario incorporar un documento semejante, en el que la persona en situación de dependencia o su familia realizara un plan de atención viable que recogiera lo que requiere la persona, con el fin de poder evaluarlo y mantenerlo actualizado para ir respondiendo a las necesidades de la persona dependiente.

\subsubsection{No hay una definición consensuada del perfil del $o$ de la asistente personal, ni una exigencia de profesionalización de las y los asistentes personales}

El Movimiento de Vida Independiente reconoce que no se exige al asistente personal que tenga gran formación en atención, cuidado o movilización, pero sí que tenga una actitud de compromiso con la persona en situación de dependencia, de respeto hacia ella, así como habilidades de comunicación, sensibilidad por las relaciones sociales; que asuma las tareas encomendadas y que las haga a gusto de la persona en situación de dependencia (Arnau y Urruticoechea, 2012). Por lo tanto, se valoran más las cualidades personales que la formación (Rodríguez-Picavea, 2007).

A este respecto, conviene aludir al currículo correspondiente al título de técnico en Atención a Personas en Situación de Dependencia, integrado en Formación Profesional de Grado Medio (Decreto 250/2012, de 27 de noviembre). En su art. 4, ("Entorno profesional”), dice así: "esta figura profesional ejerce su actividad en el sector de servicios a las personas: asistenciales, psicosociales y de apoyo a la gestión doméstica”. Y entre las ocupaciones y puestos de trabajo más relevantes, incluye la de asistente personal. En el contenido establecido para las dos mil horas de formación que recibirá el alumnado de este grado, no se hace ninguna mención a la filosofía del Movimiento de Vida Independiente.

Por otra parte, la mayoría de las empresas que tienen a sus empleadas/empleados contratados en el régimen general tienen una titulación mínima de auxiliar de geriatría, o auxiliar de clínica, y las que no (muchas personas llevan más de 25 años trabajando en el área de la ayuda a domicilio o recién incorporadas al mundo laboral) han realizado muchos cursos de formación relacionados con el área de trabajo. En acuerdos del Consejo Interterritorial, se recomienda que, para 2015, todas las/los profesionales que trabajan en servicios o centros de servicios sociales tengan acreditada la profesionalidad, bien por haber cursado estudios o por experiencia laboral documentada. Esta obligación también afecta a las personas asistentes personales, aunque actualmente no hay una fecha límite para su acreditación.

En el Estudio cualitativo del impacto de las prestaciones económicas de dependencia en el territorio histórico de Gipuzkoa (Siadeco, 2012), se señala la carencia formativa que tenían muchos trabajadores contratados como asistentes personales; y en particular, la población inmigrante que accedía a dichos empleos.

Por otro lado, las personas atendidas son mayores de 65 años en su gran mayoría (casi el 95\%), y en ocasiones son las propias familias las que enseñan a las y los asistentes personales las tareas que deben realizar. Gran parte de la población contratada directamente por las familias es inmigrante, y sus usos y costumbres son diferentes a los nuestros. Aun tratándose de una debilidad, es cierto que a la persona trabajadora se le 'adiestra' según las necesidades de la persona en situación de dependencia, por lo que las familias tienen una sobrecarga cuando contratan; pero conocido el trabajo, a la larga, ello representa una seguridad de que las tareas se realizarán como se han enseñado.

\subsubsection{Creación de un Registro de Entidades de Servicios Sociales a partir de marzo de 2013}

En julio de 2012, el Gobierno Vasco aprobó la creación de un registro de entidades que trabajan en el ámbito de los servicios sociales. El Decreto correspondiente entraba en vigor a los ocho meses de publicación y, para entonces, cada administración (foral, municipal) debía crear sus propios registros. El plazo terminó el pasado marzo y todavía no hay ni siquiera un borrador redactado en el ámbito foral. La situación es parecida en toda la Comunidad Autónoma Vasca, y el asunto se ha aplazado hasta finales de 2013.

Por un lado, hay que trabajar para regularizar las entidades que ya prestan servicios y que tienen convenios o contratos con la administración foral. Por otro, hay que marcar criterios de acceso a las empresas del sector servicios que actualmente trabajan en el ámbito de los servicios sociales, y donde el fin social o el límite en el ánimo de lucro no están claros. Éste sería el caso, por ejemplo, de las empresas que prestan servicios de asistencia personal y también realizan 'gestiones administrativas' de supuesta supervisión de las personas contratadas directamente por la persona en situación de dependencia. 


\subsubsection{Escaso seguimiento del volumen de prestaciones económicas de asistencia personal}

El seguimiento que desde los servicios sociales de base se realiza de las prestaciones económicas de dependencia suele ser escaso. Aquellas personas que disponen de servicios de ayuda a domicilio o acuden a un centro de día tienen una supervisión adicional. El resto de casos reciben un seguimiento muy general. La Sección del Departamento lleva a cabo un seguimiento administrativo (cruce de datos con los padrones, solicitud de justificación anual de gasto), pero no se hace un seguimiento de la calidad del servicio y es necesario realizarlo. En las reuniones con las personas responsables de los servicios sociales de base se ha incidido en esta prioridad, y se han enviado los datos actualizados de las prestaciones económicas que cada municipio tiene en vigor. Todos creemos necesario este seguimiento, y hay que adecuar cauces para poder realizarlo.

\subsection{Amenazas}

\subsubsection{Modelo de contratación que realiza la persona en situación de dependencia}

La prestación económica de asistencia personal conlleva la contratación de una persona para que realice las tareas que le sean encomendadas. Para llevar a cabo dicha contratación, es necesario:

1. Un contrato de trabajo. Cualquier persona puede ser contratada como asistente personal; en el caso de las personas inmigrantes, aun teniendo permiso de residencia, además, deben tener permiso de trabajo, pues de lo contrario no sería una contratación legal. El contrato de trabajo especifica las tareas, el horario, el sueldo, el número de pagas, las vacaciones y el descanso semanal y todas aquellas particularidades pactadas.

2. El alta en la Seguridad Social. Todo trabajador debe estar de alta en la Seguridad Social, condición indispensable para que el trabajo sea legal y tanto el contratante como la persona contratada estén en situación legal frente a la Administración. El alta en la Seguridad Social garantiza el pago de las cotizaciones al trabajador o trabajadora, y reconoce sus derechos (bajas, cotización para una futura pensión).

Cada una de estas modalidades de alta tiene características propias. En lo que se refiere a las empresas de servicios, cabe distinguir tres tipos de entidades:

- Empresas que se dedican a dar servicios de atención a personas en situación de dependencia. Los/las trabajadores/as pertenecen a la plantilla de la empresa y están dados de alta en el régimen general. En este caso, el contrato se realiza entre la persona en situación de dependencia y la empresa de servicios. Este tipo de empresas tienen unos costes elevados y proporcionan a la persona en situación de dependencia la seguridad de que siempre va a tener a alguien en casa que le va a atender, aunque no siempre sea la misma persona, bien porque es la empresa la que decide quién acude a cada domicilio, bien porque se requiere atención en dos tramos distintos del día (por ejemplo, dos horas por la mañana y dos por la tarde).

- Empresas de selección de personal que buscan un/a trabajador/a para desempeñar las tareas. La empresa ofrece estos trabajadores a las personas en situación de dependencia, cobra por esta selección y realiza los papeles para que la contratación se realice a través de un alta como empleada de hogar. La persona en situación de dependencia debe conocer que ella es la persona empleadora y que la relación con la empresa termina en el momento de abono por el trabajo realizado. Una vez contratada, trabajadora y persona en situación de dependencia no tienen intermediarios.

- Empresas que funcionan como gestorías. Realizan la selección del personal, dan de alta en el régimen de empleadas de hogar al trabajador o trabajadora y la persona en situación de dependencia, y todos los meses realizan el abono de la cuota de la Seguridad Social, elaboran y pagan su nómina y realizan una factura a la persona en situación de dependencia por los trámites realizados durante el mes. La empresa no tiene ninguna vinculación laboral con el trabajador o trabajadora. Este punto no siempre es conocido por la persona contratante, ya que la nómina que presenta incluye una parte referida al abono a la empresa que únicamente sirve de intermediaria para el pago a la Seguridad Social, y otra parte referida al abono de la mensualidad a la persona trabajadora.

En cuanto a la contratación directa entre la persona en situación de dependencia y el/la asistente personal, el alta en la Seguridad Social sólo es posible en el régimen de autónomos o en el de empleadas del hogar. Las funciones de una empleada del hogar, tal y como es concebida por la Seguridad Social, aparecen recogidas en el Cuadro 2.

\begin{tabular}{|l|l|}
\hline $\begin{array}{l}\text { Cuadro 2. Actividades que definen a una empleada de } \\
\text { hogar, según la Seguridad Social }\end{array}$ \\
\hline Actividades incluidas & Actividades excluidas \\
\hline - Tareas domésticas. & - Relaciones concertadas por \\
- Cuidado o atención de los & personas jurídicas, de carácter \\
miembros de la familia. & civil o mercantil. \\
- Otros trabajos, tales como & - Relaciones concertadas a \\
guardería, jardinería o & través de empresas de trabajo \\
conducción de vehículos, & temporal. \\
cuando formen parte & - Relaciones de cuidadores \\
del conjunto de tareas & profesionales contratados por \\
domésticas. & instituciones públicas o entida- \\
& des privadas. \\
& Relaciones de cuidadores no \\
& profesionales en atención \\
& a personas en situación de \\
& dependencia en su domicilio. \\
& - Relaciones concertadas entre \\
& familiares. \\
& - Trabajo a título de amistad, \\
& benevolencia o de buena \\
& vecindad. \\
\hline
\end{tabular}

Fuente: Elaboración propia a partir de Ministerio de Empleo y Seguridad Social (2013). 
Como puede observarse, entre las actividades de la empleada de hogar se encuentra la de atención de los miembros de la familia, pero se excluyen las relaciones de cuidadores no profesionales en atención a personas en situación de dependencia en su domicilio. Las funciones de una trabajadora o trabajador contratado por una persona en situación de dependencia podrían encuadrarse tanto en la primera columna del Cuadro 2 (actividades incluidas) como en la segunda (actividades excluidas). Esta contradicción ha hecho que empleados de alguna oficina de afiliación de la Seguridad Social hayan hecho una lectura restringida $y$, durante el último año, hayan cuestionando las altas de estos/as trabajadores/as en el régimen de empleadas del hogar.

Este control por parte de la Seguridad Social podía poner en cuestión todo el modelo de gestión de la prestación económica de asistencia personal, pues como se ha señalado en los datos estadísticos, el $90 \%$ de las solicitudes se realizan con contratación directa de la persona en situación de dependencia y la trabajadora. Sin embargo, a finales de octubre de 2013, la sección jurídica de las oficinas de afiliación a la Seguridad Social, ubicada en Madrid, ha realizado un nuevo encuadramiento de las/los asistentes personales y ha dado por buena el alta en el régimen de empleadas de hogar. Esta nueva situación soluciona el problema, no siendo actualmente una amenaza para los usuarios de la prestación económica de asistencia personal que deseen contratar directamente a una/un asistente personal.

\subsubsection{Continuo debate de si es sostenible el gasto en prestaciones económicas de dependencia}

El continuo cuestionamiento del gasto que suponen las prestaciones económicas de dependencia y la actual situación de crisis económica que vive el Estado llevan a recortes a escala estatal de las prestaciones. La Diputación Foral de Gipuzkoa no se ha sumado a dichos recortes, pero al ser el Estado quien finanza, en parte, dichas ayudas, la Diputación debe aportar dinero de sus propios presupuestos. Si ha habido una mengua en lo recaudado por la Hacienda Foral y no se quiere recortar en prestaciones, otros departamentos saldrán perjudicados.

Este cuestionamiento del gasto social es una amenaza desde el momento en que muchos ciudadanos ven cómo la administración pública realiza un gran desembolso en algunas áreas de atención, como las personas mayores con dependencia, mientras que otros colectivos, como los parados de larga duración o las familias desahuciadas de sus viviendas, no disponen de las mismas ayudas.

Aun siendo este punto una amenaza, porque la situación económica lleva a cuestionar el gasto social, algunos economistas (Zubiri, 2009) empiezan a plantear que la inversión en servicios sociales, y concretamente en esta prestación, redunda favorablemente en la economía, porque devuelve a la Hacienda Foral la inversión realizada a través de las declaraciones de renta de los sueldos de los trabajos realizados.

\subsection{Fortalezas}

\subsubsection{Prestación económica de derecho subjetivo}

La Ley de Autonomía y Atención a Personas en Situación de Dependencia avanza en la concepción de los derechos sociales para todos los ciudadanos, proclamando como derecho subjetivo el acceso a la cartera de servicios sociales (servicios y prestaciones) para personas en situación de dependencia. Este derecho, reconocido desde hace años en muchos países europeos, es el primer paso para reivindicar unos servicios sociales públicos para la ciudadanía. Con la aprobación de la Ley vasca de Servicios Sociales en 2008 y declararse éstos también como derecho subjetivo, se concretan el resto de las áreas de intervención de los servicios sociales y se amplía su cobertura a todos los ciudadanos que vivan en una situación de desprotección. Considerarse como derecho significa que la Administración está obligada a proporcionarlo, y el/la ciudadano/a puede exigirlo, si es necesario, en los tribunales de Justicia.

\subsubsection{Cumplimiento de los principios de la Ley $39 / 2006$}

Con la prestación económica de asistencia personal, se cumplen varios de los principios señalados en la Ley 39/2006 (art. 3i), entre otros, el de permanencia de la persona en situación de dependencia, siempre que sea posible, en el entorno en el que desarrolla su vida. No cabe duda de que la prestación de asistencia personal contribuye al cumplimiento de ese objetivo, al facilitar servicios de apoyo como los centros de día, la ayuda a domicilio, las estancias temporales o las ayudas técnicas.

\subsubsection{Consignación presupuestaria para cubrir las prestaciones económicas de dependencia}

Desde el punto de vista presupuestario, la partida económica destinada a las prestaciones económicas de dependencia no ha sufrido recortes. Como se ha visto en los datos estadísticos, el gasto en la sección de prestaciones económicas de dependencia ha ido en aumento, y aunque hay ajustes continuos en el Departamento de Política Social, los perceptores de las prestaciones económicas de dependencia no son los perjudicados.

\subsubsection{Ampliación de la prestación económica de asistencia personal a todos los grados y niveles}

Con el Decreto Foral 2/2013, de 22 de enero, que modifica el Decreto Foral 25/2009, de 21 de julio, se amplía la cobertura de atención a personas con valoración de dependencia de grado I y puntuación 
superior a 40 puntos para el acceso a la prestación económica de asistencia personal. Tiene carácter retroactivo desde el 1 de agosto de 2012.

Dicha ampliación se contemplaba en el Real Decreto Ley $20 / 2012$, de 13 de julio, de medidas para garantizar la estabilidad presupuestaria y de fomento de la competitividad, a escala estatal. Hasta el momento de la publicación de esta norma, el acceso a la prestación económica de asistencia personal estaba restringido a las personas en situación de dependencia valoradas como grandes dependientes, es decir, con grado III (más de 75 puntos).

Las cuantías económicas tienen unos límites -según el grado y puntuación de la persona en situación de dependencia- que van desde los $300 €$ a $833 €$.

\subsubsection{Interpretación amplia del art. 19 de la Ley para la Autonomía y Atención a las Personas en situación de dependencia}

A diferencia del resto del Estado, en Gipuzkoa la prestación económica de asistencia personal se concede para facilitar "el acceso a la educación y al trabajo, así como una vida más autónoma en el ejercicio de las actividades básicas de la vida diaria". Esta amplia concepción de la atención ha llevado a atender sobre todo a personas mayores (el $95 \%$ de las prestaciones son para personas mayores de 65 años). En comparación, en Gipuzkoa se conceden más prestaciones de asistencia personal que en todo el Estado junto, donde se ciñen a la atención necesaria para el acceso a la educación y al trabajo (Imserso, 2013). Esta amplia interpretación del artículo de la Ley contribuye a que la prestación económica de asistencia personal se dirija a aquellas personas que han optado por permanecer en su domicilio con diversos apoyos.

\subsubsection{Buena y amplia red de servicios sociales de base}

Gipuzkoa cuenta con 88 municipios. En todos ellos existen unidades de servicios sociales de base; algunos, por su gran tamaño, descentralizados en barrios o distritos (Donostia, Pasaia, Errenteria); y otros, centralizados en mancomunidades 0 asociación de municipios (Saiaz, Uli Mankomunitatea). La creación de estas unidades data de los años ochenta, y podemos decir que treinta años más tarde están totalmente afianzadas en sus municipios. Aunque la Ley de Autonomía y Atención a Personas en Situación de Dependencia supuso un trabajo extraordinario de información, tramitación de solicitudes (baremo de valoración de la dependencia, prestaciones económicas), significó también un acercamiento de cierta parte de la población que nunca antes había tenido relación con los servicios sociales, porque consideraban que no eran población 'necesitada'. Este 'encuentro' entre población no especialmente desprotegida y los técnicos municipales ha dado lugar, en muchas ocasiones, a trabajar más, y a hacerlo fuera de situaciones marginales o momentos de crisis. Muchos trabajadores sociales municipales valoran positivamente esta 'nueva' situación.

\subsubsection{Informatización avanzada de la tramitación de solicitudes}

La gestión de las prestaciones económicas de dependencia y, en concreto, de las relativas a las de asistencia personal, se puede decir que es dinámica, por su rapidez de resolución; moderna, porque cuenta con una aplicación informática en red que integra a todos los municipios de Gipuzkoa, y ahorra tiempo y papel; personalizada, a través de los servicios sociales de base de cada municipio de Gipuzkoa; y ágil, porque facilita los trámites del usuario y la concesión de la prestación en menos de tres meses desde su tramitación. Gracias a la tecnología informática, la situación de cada expediente se actualiza al momento y lo mismo cabe decir de la aclaración de dudas al usuario (vía telefónica o correo electrónico). Para gestionar la información, las dudas y los trámites, los servicios sociales de base y de la ciudadanía en general disponen de una dirección de correo electrónico («mendekotasuna-laguntzak@gipuzkoa.net») en la que pueden solventar en el día cualquier duda o reclamación.

\subsubsection{Pago directo}

El abono de la prestación económica de asistencia personal se realiza directamente a la persona en situación de dependencia, quien, a su vez, pagará a su asistente personal o a la empresa contratada. La persona en situación de dependencia (o su representante/familia) es la gestora de las necesidades propias y las prestaciones económicas concedidas. El abono se realiza a mes vencido, es mensual y siempre del mismo importe. A lo largo del año, se ajustan a la baja o al alta (nunca más de la cuantía tope) los gastos, según los cambios registrados en el contrato o en la utilización de servicios compatibles (servicio de asistencia domiciliaria y centros de día).

Esta posibilidad de control de la ayuda es importante, porque valora la capacidad de las personas en situación de dependencia y proporciona la autonomía suficiente para cubrir los gastos que la atención de terceras personas genera.

\subsubsection{Justificación anual del gasto}

En la justificación del gasto de 2011, las solicitudes en las que se detectaron irregularidades (bajas en la Seguridad Social, cambio de trabajadores sin haberlo comunicado, traslados a otras provincias del Estado y bajas en padrón) no llegaron al 10\%. La justificación del gasto de 2012 ha tenido una respuesta muy buena, siendo inferior al $5 \%$ las irregularidades detectadas. 
Una vez al año (en marzo, habitualmente) se envía una carta a todas las personas que perciben la prestación económica de asistencia personal para que justifiquen los gastos del año anterior. Estas justificaciones se realizan desde 2010, año en que el volumen de prestaciones concedidas era ya muy elevado (se acercó a las mil personas con prestación) y no se podía realizar de oficio. En las justificaciones de 2011 y 2012 , se contabilizaron un $10 \%$ y menos de un $5 \%$, respectivamente, de personas que habían cometido algún tipo de 'fraude', al cobrar la prestación sin tener personal contratado con los requisitos exigidos. Obtener una respuesta del 90-95\% de las solicitudes se valora como muy positivo, porque constata la madurez de la ciudadanía en el cumplimiento de los requisitos y el uso de las prestaciones públicas.

\subsection{Oportunidades}

5.4.1. Modificación del Decreto Foral actual, de forma que contemple un desarrollo normativo mayor en la prestación económica de asistencia personal

La ampliación de la cobertura a otros grados es un paso más en la atención a personas en situación de dependencia. Esto debería llevar también a regular mejor dicha prestación, a través de un nuevo decreto foral sobre las prestaciones económicas de dependencia en el que se regulen aspectos que tienen que ver con la contratación de las/los asistentes personales, la formación, el seguimiento de las prestaciones por parte de las unidades de servicios sociales de base o la elaboración de planes de atención individual, entre otras cuestiones.

\subsubsection{Voluntad política de impulsar, desarrollar y potenciar la prestación económica de asistencia personal}

La voluntad del actual equipo de Gobierno del Departamento de Política Social y, en conjunto, de la Diputación Foral de Gipuzkoa, de mantener los niveles de cobertura en servicios y prestaciones de Servicios Sociales es clara ${ }^{4}$. El de Política Social es el único presupuesto de todos los departamentos que no ha sufrido un descenso de su presupuesto global, aunque sí una reorganización de éste.

En concreto, se ha optado por ampliar la cobertura extensa de la prestación económica de asistencia personal a todas las personas con un nivel de dependencia mínimo de grado I y 40 puntos, cuando a escala estatal la prestación se restringe a personas con grado II y 50 puntos, como mínimo. Las cuantías económicas son un $15 \%$ superiores a la media del

4 En los números de Gipuzkoa Berria -publicación divulgativa buzoneada por la Diputación Foral de Gipuzkoa- editados en primavera de 2013 y noviembre de 2013 , se hace mención a la voluntad política del Gobierno de la Diputación Foral de Gipuzkoa de desarrollar las prestaciones económicas para personas en situación de dependencia.
Estado (cada comunidad autónoma tiene la potestad de establecer la cuantía máxima que desee).

\subsubsection{Aumento de solicitudes de prestaciones económicas de asistencia personal y migración de las prestaciones económicas de cuidado en el entorno familiar}

Hasta noviembre de 2013, se han registrado 923 nuevas solicitudes de prestación económica de asistencia personal, de las cuales, 310 son 'migraciones' de prestaciones económicas de cuidado en el entorno a prestaciones económicas de asistencia personal. A veces, esto se realiza para formalizar una contratación que ya existía anteriormente, aunque la familia estuviera atendiendo también a la persona en situación de dependencia. En otros casos, esto ha servido para visibilizar la atención asistencial que ya tenían personas con grado II o grado I (con 40 puntos). Es objetivo del Departamento que aumenten las prestaciones económicas de asistencia personal y disminuyan las de cuidado en el entorno.

\subsubsection{Creación de empleo o regularización de las contrataciones ya existentes (altas en Seguridad Social)}

El sector de la asistencia personal es un yacimiento de empleo, o cuando menos, una oportunidad de regularizar contrataciones ilegales que existen en algunos domicilios y, por tanto, de regular la situación de inmigrantes (que representan el $70 \%$ de las contrataciones realizadas directamente por la persona en situación de dependencia).

La prestación económica de asistencia personal obliga a las personas trabajadoras a tener un contrato legal y estar de alta en la Seguridad Social, condiciones sin las cuales no es posible recibir la prestación económica. Esta situación redunda en el beneficio la persona empleadora y la contratada.

\subsubsection{Visibilidad del trabajo de atención personal en el domicilio, a través de las solicitudes de prestación económica}

Es importante también visibilizar la atención de las personas en situación dependencia que se quedan en sus hogares, y verlas no sólo como gasto, sino como generadoras de servicios y trabajo; es decir, un gasto que revierte en la sociedad a través de contrataciones, seguros sociales o pago de impuestos. La opción de permanecer en el domicilio ha estado supeditada a tener los suficientes apoyos económicos y familiares. Actualmente es una opción más, pero la permanencia en el domicilio conlleva atención, cuidado, supervisión y seguimiento de la persona en situación de dependencia, así como del cumplimiento de la atención que necesita. 


\subsubsection{Perspectiva de género en la atención de las personas en situación de dependencia}

Como indican los datos estadísticos, el 94,5\% de las personas asistentes personales en 2013 son mujeres. Alguien podría decir que este factor no es una oportunidad, sino más bien una debilidad, porque seguimos manteniendo el rol femenino en tareas que tienen que ver con el cuidado doméstico y la atención a personas en situación de dependencia. Es cierto y esto se constata. También es cierto que esta realidad tiene que verse, que quedar en evidencia, porque si no, nunca se va a poder intervenir en este colectivo, que además de ser mujer, es inmigrante (70\%) y cobra sueldos 'mileuristas'. Las estadísticas no pueden quedarse sólo en porcentajes en un papel, sino que deben provocar una reacción, por lo menos, para que se visualice.

\section{Entrevista a empresas proveedoras de servicios de asistencia personal}

Para conocer de cerca algunas de las empresas que proveen servicios de asistencia personal, se entrevistó a once de ellas. En concreto, se quería indagar en su funcionamiento, en cómo se establecía la relación con las personas usuarias y en qué servicios ofrecían, entre otros aspectos. La entrevista se basó en un cuestionario elaborado ad hoc, que constaba de tres partes: ficha de datos generales, datos de la empresa y datos de las/los asistentes personales.

Las entrevistas se realizaron entre el 3 de mayo y el 13 de mayo de 2013 , con cita previa, en la oficina de la entidad en cuestión. La elección de las empresas tenía como objeto elegir aquellas que tuvieran modelos distintos de gestión tanto del personal (contrataciones en régimen general o no) como del servicio domiciliario. De once entrevistas, nueve se realizaron a empresas de servicios que tenían varios clientes con concesión de prestación económica de asistencia personal, y otras dos, a empresas que han iniciado su andadura en 2013 y aportaban novedades en la atención personal (Bizikide y Matia Fundazioa). Las empresas visitadas fueron: Arreta Zerbitzuak, Adas, Soluciones Loreka, Landu, Matia Fundazioa y Bizikide (Donostia); Etxekoi Bidasoa y Zenturia (Irun); Ilarki (Tolosa); Hamabi T (Pasaia); y Konfia (Lasarte-Oria).

\subsection{Resumen de los datos obtenidos en las visitas a empresas}

- Salvo Adas y Matia Fundazioa, el resto de empresas ofrecían, además de servicio de ayuda a domicilio (doméstico y de atención personal), otros servicios de limpieza, guardería, gestoría y selección de personal.

- Excepto Soluciones Loreka, que no tiene personal propio en asistencia domiciliaria, las demás tienen todo su personal dado de alta en el régimen general de la Seguridad Social. Aunque el número de trabajadores contratados sea alto, llama la atención que no tengan jornadas de trabajo completas. El motivo principal es que la atención a las personas se realiza, en su mayoría, a primeras horas del día y que se tiene muy en cuenta el perfil que la familia solicitante, por lo que a veces se contrata a trabajadoras para un único servicio y de pocas horas.

- Los trabajadores que desempeñan su labor como asistentes personales en los domicilios tienen el convenio de ayuda a domicilio estatal con mejoras, excepto la empresa Konfia, que posee convenio propio, y Hamabi T, que se acoge al convenio de limpieza estatal, más alto que el de ayuda a domicilio (en sueldo y tres pagas extra).

- Las empresas que realizan gestiones (nóminas, altas y bajas en la Seguridad Social) y cobran mensualmente por este servicio realizan un contrato con las personas usuarias, a quienes se les cobra en función del coste que tienen con su empleada de hogar. Las tareas de gestión -y los costes correspondientes- varían: en algunos casos, sólo incluyen gestoría (Konfia), y en otros, se realiza un seguimiento de los trabajadores en los domicilios (Zenturia, Bizikide, Soluciones Loreka). Según las empresas, resulta claro que estos servicios de gestoría no forman parte intrínseca del servicio de atención domiciliaria, pero que las familias perciban realmente esa diferencia resulta cuestionable.

- Los trabajadores están titulados en Auxiliar de Clínica o Auxiliar de Geriatría, o han recibido cursos formativos en personas mayores. Se realizan cursos de reciclaje organizados por la propia empresa (Matia Fundazioa e Ilarki) o impartidos por otros organismos, como Sartu u Hobetuz. En estos casos, la asistencia no es obligatoria, salvo en la empresa Konfia, donde la formación se imparte además en horario de trabajo.

- En todas las empresas, las plantillas están formadas mayoritariamente por mujeres. En casi todas, hay uno o dos trabajadores varones, excepto en Adas, donde éstos constituyen un tercio del personal (diez trabajadores sobre treinta). La edad ronda los 40045 años, y además las personas atendidas demandan que las y los asistentes sean de mediana edad, porque les da seguridad. Hay casos en que, aun siendo jóvenes, las asistentes han sido bien aceptadas por su vitalidad y buen trato.

- El colectivo inmigrante va perdiendo peso en los dos últimos años, sobre todo porque cada vez hay más personas autóctonas que quieren realizar atención domiciliaria y porque muchos usuarios informan a la empresa de que no quieren inmigrantes. Las personas inmigrantes que están trabajando llevan una media de tres años y están totalmente integradas en la empresa. Landu y Konfia son las empresas que más inmigrantes tienen en plantilla. Las trabajadoras de Konfia pertenecen al grupo de personas en procesos de inserción social. 
- Pocas empresas realizan planes de atención, únicamente Matia Fundazioa y Konfia.

- Otras empresas, en concreto las que tienen adjudicación del servicio de asistencia domiciliaria municipal (Hamabi T, Arreta Zerbitzuak e Ilarki), tienen conocimiento de los planes de atención, porque son realizados en los ayuntamientos, pero no los utilizan como instrumento de trabajo. En Bizikide, que atiende a las personas tuteladas de Fundación Hurkoa, también saben de la existencia de planes de atención, pero no están integrados en su trabajo.

- La demanda de trabajadores con conocimiento de euskera varía según las zonas. No se solicitan en la zona de Irun, pero sí en Tolosaldea y Hondarribia. La empresa Ilarki realiza todos los años un curso de reciclaje de euskera para sus trabajadoras. Ante una demanda de los usuarios, todas las empresas responden que tienen en cuenta la petición y buscan a una persona con perfil de euskera.

- La supervisión continua de casos se lleva en las empresas que tienen la adjudicación del servicio de asistencia domiciliaria municipal (Arreta Zerbitzuak, Ilarki y Hamabi T, Matia Fundazioa y Konfia). En el resto, se realiza seguimiento muy general del caso.

- Todas las empresas reciben currículos de personas que desean trabajar en ellas, excepto Matia Fundazioa, que se nutre de su propio colectivo de trabajadoras. La gran mayoría llegan de currículos por correo electrónico. El número recibido diariamente es abrumador, por lo que la mayoría solicita que se envíen por la citada vía. Algunas empresas (Etxekoi Bidasoa, Konfia, Soluciones Loreka y Adas) seleccionan currículos y realizan entrevistas todas las semanas.

- El personal que dirige las empresas, en su mayoría, pertenece al perfil de gestión económica en Empresariales, con formación en recursos humanos. Sólo Arreta Zerbitzuak, Konfia, Adas y Matia Fundazioa tienen personal técnico con titulación en Trabajo Social o Psicología. Ilarki, Etxekoi Bidasoa y Zenturia, por su parte, están dirigidas por personal titulado en Auxiliar de Clínica o Geriatría, y formación en administración.

- Pocas empresas se han dedicado siempre a la asistencia domiciliaria; la mayoría provienen del mundo de la limpieza en empresas, portales y domicilios, así como de los servicios de conciliación familiar. Las empresas que siempre se han dedicado a atención a personas en situación de dependencia son Adas, Matia Fundazioa, Ilarki, Hamabi Ty Landu.

- Respecto a la profesionalización, todas las empresas coinciden en que la atención domiciliaria tiene fama de que cualquiera puede realizarla y de que ir a un domicilio es sencillo. Creen necesario reivindicar la formación y que se exija una formación mínima a toda persona que realice atención domiciliaria. Las empresas aseguran que los trabajadores están formados y algunas exigen a la administración pública (en este caso, a la Diputación Foral) que controle y supervise la atención que se realiza en domicilios con personal contratado directamente. Saben que a mayor número de horas de atención, a las familias les sale más rentable contratar a alguien directamente, pero en muchas ocasiones no son personas con formación. A este respecto, la empresa Soluciones Loreka, que realiza selección de personal y no tiene asistentes personales en nómina, hace un seguimiento en los casos en que la familia lo solicita. Así, además de realizar las gestiones de nóminas, altas y bajas de la Seguridad Social, realiza encuestas de satisfacción al usuario y a la persona trabajadora. Bizikide también lleva a cabo este tipo de seguimiento, y media cuando hay situaciones conflictivas entre las partes.

- Adas menciona que la asistencia personal mediante trabajadoras internas en un domicilio no forma parte de su filosofía. Considera que este tipo de atención no puede ser buena; que para atender las veinticuatro horas son necesarios tres trabajadores, y que si se trata de atender los siete días de la semana, esa cifra subiría a cinco, lo que resultaría insostenible económicamente. Aboga por que los trabajadores sean profesionales, que, en unas ocasiones, atenderán casos 'sencillos', y en otras, situaciones más complicadas -inicios de enfermedad de Alzheimer, enfermos mentales, personas con mucho carácter, casos de intromisión de otros miembros de la familia-, y para todas las situaciones hay que estar preparado y formado.

\section{Conclusiones del diagnóstico del modelo de gestión}

Después de casi seis años de andadura, la redacción de dos decretos forales y en espera de que se elabore el tercero, es necesario reflexionar sobre la gestión actual de la prestación económica de asistencia personal y proponer mejoras a fin de que las personas en situación de dependencia puedan optar a ella para apoyar su permanencia en el domicilio y que ésta sea óptima. Éste es el objetivo de la prestación y también de quienes trabajamos para que esto se cumpla.

Realizar el diagnóstico de la gestión actual ha supuesto un trabajo de ordenar ideas. Cuando se está involucrado totalmente en la práctica, es difícil poner distancia y mirar lo que se hace como si no estuvieras dentro. Ni todo lo que se hace está bien, ni todo es un desastre irremediable.

Podríamos decir que el modelo actual goza de una buena salud: hay puntos fuertes, las fortalezas son firmes y dan continuidad a la gestión. La mejora es un camino a largo plazo; no hay visos de que estemos ante una situación que se tambalee. Damos respuesta al ciudadano con una prestación que la Ley estatal de Promoción de la Autonomía y Atención a las personas en situación de dependencia y la Ley autonómica de Servicios Sociales promueven como derecho subjetivo. 
Cumplimos el objetivo de que la persona en situación de dependencia permanezca en el entorno en el que vive. Hay consignación presupuestaria para cubrir el abono mensual de las prestaciones económicas de dependencia y las solicitudes se van aprobando en un plazo inferior a los tres meses desde su solicitud. Tenemos una buena red de servicios sociales de base municipales y la relación con la Diputación Foral es estrecha. La tramitación de las prestaciones se realiza con agilidad, al contar con un sistema informático que facilita las gestiones al usuario y ha desterrado casi en su totalidad el papel. La cobertura de las prestaciones no sólo en el acceso a la educación y al trabajo, sino también a una vida más autónoma en el ejercicio de las actividades básicas de la vida diaria, ha facilitado que se atienda al colectivo de personas mayores, que constituyen el $95 \%$ de quienes han solicitado esta prestación. Que se conceda esta ayuda a la persona en situación de dependencia y que sea ella quien la gestione es un hecho importante, por lo que dice de la responsabilidad y autogestión de sus propias necesidades, y a la vez, de dar cuentas de los gastos a la administración pública. En este sentido, sólo un $10 \%$ de las prestaciones económicas de asistencia personal concedidas en 2011 y un $5 \%$ de las aprobadas en 2012 no tuvieron una correcta justificación durante todo el año. Dándole la vuelta al dato, que el 90-95\% sí lo justificara constata la madurez en el cumplimiento de las obligaciones ciudadanas respecto a la Administración.

Si podemos sentirnos orgullosos de las fortalezas de este modelo, también hay que decir que tenemos 'problemas' que deben ser atajados para que no se abran grandes brechas. En este sentido, la situación actual de crisis y el gran gasto social que se realiza hoy día son una amenaza importante. El continuo cuestionamiento de si es posible mantener este gasto también va calando en la población, y este discurso hay que contrarrestarlo, tomando el gasto social de las prestaciones económicas de dependencia como una oportunidad para crear empleo. Nuestro modelo también tiene debilidades: falta un plan de atención individual de lo que la persona en situación de dependencia precisa, es necesario un seguimiento de la calidad del servicio prestado, no hay un perfil declarado de funciones del asistente personal, y hay poco desarrollado en el campo formativo. Considero que a estas debilidades sí les podemos hacer frente, que muchas están en nuestra mano y que hay que reglamentar para que se solucionen. De las oportunidades, cabe comentar la posible aprobación de un nuevo decreto que servirá para trabajar en mejorar el modelo actual y asegurar su continuidad en el tiempo.

Respecto a las entrevistas a empresas que ofrecían servicios domiciliarios de atención, fue interesante conocer qué empresas trabajaban en este campo. Las prestaciones económicas de asistencia personal gestionadas a través de empresas sólo suponen un $10 \%$, pero que hubiera 22 empresas en 2012 y que en 2013 se hayan creado 8 nuevas supone una gran proliferación en un sector que carece de reglamentación. Muchas se dedican a la asistencia personal desde hace años y tienen adjudicados los servicios de ayuda a domicilio de los municipios de Gipuzkoa. Otras se han dedicado a la atención en domicilios con demanda privada; otros tantas, a la selección de personal y la gestión de nóminas y altas y bajas de la Seguridad Social, y al seguimiento. Se trata de cuestiones que es necesario regular, por el desconocimiento que suponen a la ciudadanía y el poco control existente respecto a lo que se hace o no.

El balance general de las visitas ha sido muy bueno. Las empresas colaboraron en todo momento. En la mayoría, se vio gran profesionalidad, preocupación por el futuro, por el desconocimiento que tienen las familias sobre las situaciones que tienen en casa 0 su incapacidad de atenderlas. Las mismas empresas exigen reglamentación. Se constató que tienen a sus trabajadores de alta en el régimen general, que todos poseen formación (rama sanitaria, geriátrica). Creen necesario que se valore el trabajo de atención a personas con dependencia, y que para ello hay que estar preparado, que no vale que cualquiera haga de todo.

\section{Propuesta de mejora de la gestión de la prestación económica de asistencia personal}

El objetivo principal de la prestación económica de asistencia personal es facilitar el mantenimiento de las personas con dependencia en su entorno habitual a través de la contratación de personas para su atención personal. Vista la demanda de solicitudes, el gasto general en prestaciones económicas de dependencia y la contratación de personal en este concepto, bien podríamos decir que vamos por el buen camino, pero es necesario dar soluciones a problemas que van surgiendo. Teniendo en cuenta el diagnóstico DAFO y las entrevistas realizadas a empresas que proveen servicios de asistencia personal, esta propuesta de mejora abarca cinco cuestiones:

- Modelo ideal de gestión de la prestación económica de asistencia personal.

- Formación básica exigible a las personas que realizan tareas de asistencia personal.

- Elaboración de un plan de atención individual.

- Elaboración de criterios para la formalización de un Registro de Empresas Proveedoras de Servicios de Asistencia Personal.

- Seguimiento de las prestaciones económicas de asistencia personal por parte de la Diputación Foral y los servicios sociales de base

\subsection{Propuesta de modelo ideal de gestión de la prestación económica de asistencia personal}

\subsubsection{Persona en situación de dependencia}

- Destinatarios:

- Toda persona valorada con un nivel de dependencia de grado I, II o III. 
- Edad: no habría edad establecida (desde el nacimiento a cualquier edad).

- Requisitos:

- Empadronamiento en cualquier municipio de Gipuzkoa.

- Presentación de un plan de atención individual elaborado por la persona en situación de dependencia (personas mayores, personas con discapacidad / diversidad funcional) 0 su familia, con la ayuda del profesional de referencia de los servicios sociales de base. En dichos planes, se especificaría si se convive con la familia o no, a los efectos de determinar la obligación de alimentos entre parientes (art. 142 del Código Civil: sustento, habitación, vestido y asistencia médica) y gastos compartidos con éstos.

- Obligaciones:

- Hacer uso de las prestaciones económicas de asistencia personal percibidas para el fin con el que han sido concedidas.

- Informar de cualquier cambio realizado en el servicio o respecto a la situación de la persona dependiente.

\subsubsection{Administración: Departamento de Política Social}

- Se constituiría un equipo técnico de valoración social y económica que evalúe los planes de atención individual según el proyecto de vida de cada persona.

- Se tomarían en cuenta los planes de atención individual y las actividades previstas, según la edad, buscando la equiparación con personas semejantes de su entorno.

- Se realizaría una supervisión y seguimiento anual de las prestaciones económicas de asistencia personal por parte de la Sección del Dpto. de Política Social y los servicios sociales de base municipales.

- Se establecería un presupuesto para dar cobertura a las personas que soliciten la prestación económica de asistencia personal.

- Respecto a la concurrencia de servicios, la prestación de asistencia personal sería compatible con el servicio de asistencia domiciliaria, con el resto de servicios sociales -centros de día, talleres ocupacionales, ayudas técnicas, estancias temporales (puntuales y justificadas) - y con la actividad laboral (empleo protegido o no).

- Se plantean tres niveles distintos de atención -servicio de asistencia domiciliaria, servicio de asistencia personal y Programa de Vida Independiente-, según la intensidad y el programa de atención individual diseñado.

\subsubsection{Tercer sector}

- La contratación de personas para realizar funciones de asistencia personal se llevaría a cabo de dos maneras:

a. a través de empresas inscritas en el Registro de Empresas de Servicios de Atención Personal; o

b. directamente, mediante contratación individual con alta en la Seguridad Social.

- El personal contratado debería tener formación en personas en situación de dependencia.

- Las empresas se encargarían de aplicar los planes de atención individual elaborados.

\subsection{Propuesta sobre la formación básica exigible a las personas que realizan tareas de asistencia personal}

La trabajadora o trabajador que acude a un domicilio como asistente personal y pertenece a una empresa debería tener formación básica en atención a personas dependientes, bien un grado en Auxiliar de Clínica o Auxiliar geriátrico, bien módulos formativos realizados en este campo. De la misma forma, asistentes personales contratadas por personas en situación de dependencia deberían cumplir con el requisito de tener conocimientos en materia de atención a personas en dependencia. Hoy día la gran mayoría de trabajadoras contratadas directamente por las personas en situación de dependencia no cuentan con dicha formación. Esta carencia se suple con la buena disposición de las/los trabajadores, motivada en parte por la necesidad de tener un trabajo y, en el caso de las/los extranjeros, de regularizar su situación de residencia.

Las personas que quieren trabajar en el campo de asistencia personal y precisen formación podrían obtenerla a través de entidades como Hobetuz o Sartu, o mediante los ciclos formativos de Lanbide. No es necesaria una gran especialización, pero sí conocimientos básicos en comunicación, actitudes, valores éticos, movilizaciones, alimentación, enfermedades relacionadas con la dependencia y buenas prácticas.

El sector que trabaja en asistencia personal debería profesionalizarse para garantizar una buena atención. Dicha profesionalización no está reñida con el modo concreto de hacer las tareas y con que éste lo dicte la persona en situación de dependencia o su familia. La persona en situación de dependencia con buen nivel cognitivo - o, en su ausencia, la familiadebe ser quien establezca quiere que se realice su cuidado y atención.

\subsection{Propuesta para la elaboración de un plan de atención individual}

La solicitud de una prestación económica de asistencia personal resulta incompleta si no se conocen las necesidades de atención que precisa la persona en 
situación de dependencia. Por ello es preciso que la propia persona, o su familia, realice un plan de atención en el que se recojan los datos básicos de identificación, y se constaten las necesidades que tiene y los apoyos que requiere para el mantenimiento en su entorno familiar y social.
En la Figura 1 se presenta una propuesta de plan de atención individual que debería presentarse junto con la solicitud de prestación económica y el resto de documentación (contrato, alta en la Seguridad Social y datos de identificación de la persona asistente personal).

\section{Figura 1. Propuesta de plan de atención individual de la persona en situación de dependencia}

\begin{tabular}{l|l} 
Persona en situación de dependencia & Asistente personal \\
Nombre y apellidos: & Nombre y apellidos: \\
DNI: & DNI o NIE: \\
Fecha y lugar de nacimiento: & Fecha y lugar de nacimiento: \\
Domicilio: & Domicilio: \\
Teléfono: & Teléfono:
\end{tabular}

Datos del baremo de valoración de dependencia

Grado:

Nivel:

Puntos:

Utilización de ayudas técnicas:

Nivel cognitivo:

Servicios y prestaciones

Centro de día:

Laboral:

Continuo:

Fines de semana:

Servicio de asistencia domiciliaria

Teleasistencia

Prestaciones económicas

Estancias temporales

Convivencia con otros miembros de la familia

Cónyuge:

Hijos/as:

Otros:

Persona cuidadora familiar:

Tareas asumidas por los miembros de la familia de convivencia:

Actividades ocupacionales o laborales que realiza la persona solicitante:

Tareas asumidas por el/la asistente personal*

TAREAS DEL ÁMBITO DOMÉSTICO

Comida

Limpieza de la casa (habitación,

baño, cocina, áreas comunes)

Recados, compras, gestiones

\section{TAREAS DE ATENCIÓN PERSONAL}

Aseo personal

Transferencias

(cama, silla de ruedas, grúa)

Cambios posturales

Acompañamiento

Salidas a la calle (paseo, gestiones)

Realizado por:

Presentado para (prestación económica de asistencia personal, nuevo plan de tareas):

Lugar y fecha:
HORARIO DE TRABAJO**

Organización del conjunto de los cuidados:

Horario de descanso (en caso de ser interno/a):

Periodo de vacaciones anual:

* Pueden presentarse en forma de cuadro de actividades y horarios.

** Estimación del número de horas de atención que se requieren para cubrir las necesidades.

Fuente: Elaboración propia. 
8.4. Propuesta de criterios para la formalización de un Registro de Empresas Proveedoras de Servicios de Asistencia Personal

El Decreto del Parlamento Vasco 155/2012, de 24 de julio, de Registros de Servicios Sociales, en su exposición de motivos, dice que "los órganos forales de los territorios históricos podrán efectuar una regulación de sus respectivos registros forales de servicios sociales de carácter complementario de la regulación de los registros que establezca el Gobierno Vasco". $\mathrm{El}$ art. 2 especifica que "todas las entidades públicas y privadas de servicios sociales y todos los servicios y centros dependientes de las mismas que actúen en el territorio de la Comunidad Autónoma de Euskadi, formen parte o no del Sistema Vasco de Servicios Sociales, deberán ser objeto de registro" y diferencia conceptualmente entre:

- Entidad de servicios sociales: "toda persona física o jurídica legalmente constituida, de naturaleza pública o privada, con o sin ánimo de lucro, que sea titular de uno o varios servicios o centros de servicios sociales, así como aquella que, careciendo de los mismos, realice actividades orientadas exclusivamente a la prestación de información con carácter general o al apoyo mutuo de sus integrantes".

- Servicio de servicios sociales: "toda actividad organizada técnica y funcionalmente, de carácter general o especializado, prestada, con carácter regular y permanente, por una entidad de servicios sociales, sin que dicha prestación deba ofrecerse necesariamente en un centro".

- Centro de servicios sociales: "todo inmueble o parte de inmueble constituido como unidad orgánica y funcional, con ubicación autónoma e identificable, en la que se ofrecen, o desde la que se articulan, prestaciones de servicios sociales".

El decreto de Gobierno Vasco entró en vigor el pasado marzo, y la Diputación Foral de Gipuzkoa todavía no ha regulado su Registro de Entidades de Servicios Sociales.

Como se puede comprobar, las empresas que proveen servicios de asistencia personal no tienen ninguna regulación más allá de las que la Hacienda Foral o la Seguridad Social exigen para la realización de una actividad. Por lo tanto, es imprescindible regular estas empresas, para lo cual se proponen los siguientes criterios de homologación:

- Las empresas proveedoras de servicios de asistencia domiciliaria deberán estar dadas de alta en el impuesto de actividades económicas en la agrupación 95 (asistencia y servicios sociales), en los grupos 9510952.
- Los trabajadores de la empresa estarán incluidos en el régimen general de la Seguridad Social. Los contratos de trabajo corresponderán con las funciones de atención personal y doméstica a las personas en situación de dependencia y se ceñirán a los requisitos exigidos en el régimen general (por ejemplo, no habrá personas internas en domicilios con horarios superiores a cuarenta horas semanales).

- Los trabajadores tendrán una formación básica como auxiliares de clínica o de geriatría, o una formación mínima de cien horas en cursos de atención a personas en situación de dependencia.

- Las empresas presentarán todos los servicios que pudieran prestar en materia de asistencia domiciliaria, tanto si se desarrollan en el domicilio como si se trata de servicios de gestión.

- Los precios establecidos por la empresa serán públicos, y se informará de la exención o no del IVA y del porcentaje aplicado.

- La empresa estará obligada a llevar a cabo el plan de atención individual previsto para atender a la persona en situación de dependencia.

- La empresa elaborará un registro mensual de las personas atendidas, el número de horas mensuales, y del tiempo dedicado a atención doméstica y atención personal.

\subsection{Propuesta para el seguimiento de las} prestaciones económicas de asistencia personal por parte de la Diputación Foral y los servicios sociales de base

Para dar respuesta a la demanda de control y supervisión de las personas en situación de dependencia que tienen asistentes personales que les atienden, se propone realizar un seguimiento, por lo menos una vez al año, a todos los usuarios beneficiarios de la prestación económica de asistencia personal. Dicho seguimiento se realizaría en colaborativamente entre la Diputación Foral y los servicios sociales de base municipales. Para ello, se tendría en cuenta: a) el plan de atención individual presentado en la solicitud de prestación económica de asistencia personal; y b) la encuesta de satisfacción realizada al usuario.

El objetivo del plan individual de atención es mantener a la persona en situación de dependencia en su entorno todo el tiempo que sea posible, con los servicios y prestaciones a los que tuviera derecho. Para valorar si dicho objetivo se cumple en la prestación de asistencia personal, se revisaría y actualizaría el plan de atención individual junto con el usuario o su familia. Como complemento a esta revisión, se valoraría la necesidad de conocer la satisfacción del usuario o su familia respecto a la atención recibida; para ello, se propone el formulario de la Figura 2. 
Figura 2. Propuesta de documento para el seguimiento de las prestaciones de asistencia personal por parte de la Diputación Foral y los servicios sociales de base: indicadores para conocer la satisfacción del usuario

\section{ÁmBITo domÉstico}

Enumerar las tareas que realiza y puntuar el nivel de satisfacción (de o a 10)

Limpieza (casa, baño, áreas comunes)

Comida (elaboración, variedad)

Cumplimiento de los horarios de trabajo

Compras, gestiones

\section{ÁmBito de ATENCIÓN PERSONAL}

Enumerar las tareas que realiza y puntuar el nivel de satisfacción (de o a 10)

Administración de comida y medicación

Aseo personal (valorar la intimidad, el respeto)

Horarios: levantarse, desayuno/comida/

merienda/cena, acostarse

\section{ASISTENTE PERSONAL}

Enumerar las tareas que realiza y puntuar el nivel de satisfacción (de o a 10)

Comunicación: ¿mantiene conversaciones cuando está con la persona en situación de dependencia?, ¿habla poco, habla mucho, respeta las opiniones de los demás?

¿Tiene un carácter amable, respetuoso, empático?

¿Tiene capacidad para improvisar ante urgencias? ¿Es resolutiva?

¿La trabajadora o el trabajador mantiene una buena relación con la persona en situación de dependencia?:

relación simétrica, relación asimétrica (asistente personal por encima de la persona en situación de dependencia o viceversa)

Desde la última supervisión, enumerar el número de personas que han trabajado como asistente personal. En caso de bajas, comentar los motivos:

Tras la experiencia, ¿continuaría con la atención de un/a asistente personal?:

Realizado por:

Realizada con (persona dependiente, familiar):

Fecha:

Fuente: Elaboración propia. 
(2013): “Decreto 250/2012, de 27 de noviembre, por el que se establece el currículo correspondiente al título de Técnico en Atención a Personas en Situación de Dependencia”, Boletín Oficial del País Vasco, no 24, 4-2-2013 [khttp://www. lehendakaritza.ejgv.euskadi.net/r48-bopv2/ es/bopv2/datos/2013/02/1300642a.pdf)].

(2013): “Decreto Foral 2/2013, de 22 de enero, de modificación del Decreto Foral 25/2009, de 21 de julio, por el que se regulan las prestaciones económicas de la Ley 39/2006, de 14 de diciembre, de Promoción de la Autonomía Personal y Atención a las personas en situación de dependencia", Boletín Oficial de Gipuzkoa, no 17, 25-1-2013 [<https://ssl4.gipuzkoa.net/ castell/bog/2013/01/25/c1300710.pdf〉].

(2013): Gipuzkoa Berria, noviembre.

(2013): Gipuzkoa Berria, primavera.

(2012): "Decreto 155/2012, de 24 de julio, de Registros de Servicios Sociales", Boletín Oficial del País Vasco, no 150, 2-8-2012 [<http://www.euskadi. net/cgi-bin_k54/ver_c?CMD $=$ VERDOC\&BASE $=$ B03A\&DOCN $=000110765 \& C O N F=/$ config $/ \mathrm{k} 54 /$ bopv_c.cnf〉].

(2012): “Real Decreto-ley 20/2012, de 13 de julio, de medidas para garantizar la estabilidad presupuestaria y de fomento de la competitividad", Boletín Oficial del Estado, n- $168,14-7-2012$, págs. 50.428 50.518 [rhttp://www.boe.es/buscar/doc. php?id=BOE-A-2012-9364'].

(2009): “Decreto Foral 25/2009, de 21 de julio, por el que se regulan las prestaciones económicas de la Ley 39/2006, de 14 de diciembre, de Promoción de la Autonomía Personal y Atención a las personas en situación de dependencia", Boletín Oficial de Gipuzkoa, n- 144, 4-8-2009, pág. 2 [rhttp://ssl4.gipuzkoa.net/boletin/ asp/ViewRoot.asp?Action $=\mathrm{Html}$ \&ltem $=4$ $\& X=1119181837>]$.

(2008): “Decreto foral 87/2008, de 23 de diciembre, por el que se regula la concesión de las ayudas individuales del Programa Etxean dirigidas a personas con discapacidad o en situación de dependencia”, Boletín Oficial de Gipuzkoa, no 251, 31-12-2008, pág. 40.595 ['https://ssl4. gipuzkoa.net/boletin/asp/ViewRoot.asp?Actio $n=H$ tml\&Item $=0 \& X=1204112251\rangle]$.

(2008): "Ley 12/2008, de Servicios Sociales del País Vasco", Boletín Oficial del País Vasco, 꾸 246, 24-12-2008, págs. 31.840-31.924 [khttp://www.euskadi.net/ bopv2/datos/2008/12/0807143a.pdf〉].
(2007): "Real Decreto 504/2007, de 20 de abril, por el que se aprueba el Baremo de Valoración de la Situación de Dependencia establecido por la Ley 39/2006, de 14 de diciembre, de promoción de la autonomía personal y atención a las personas en situación de dependencia”, Boletín Oficial del Estado, no 96, 21-4-2007, págs. 17.646-17.685 [khttp://www.boe.es/ buscar/doc.php?id=BOE-A-2007-8350>].

(2006): “Ley 39/2006, de 14 de diciembre, de Promoción de la Autonomía Personal y Atención a las personas en situación de dependencia", Boletín Oficial del Estado, ํㅜ 299, 15-12-2006, págs. 44.142-44.156 [<http://www.boe.es/boe/ dias/2006/12/15/pdfs/A44142-44156.pdf>].

ARNAU, S.; y URRUTICOECHEA, B. (2012): “¿Hablamos de formación o de qué?”, en HIDALGO LAVIÉ, A. (coord.), Trabajo social en defensa de la asistencia personal. Reflexiones y sugerencias, Madrid, Universidad Nacional de Educación a Distancia; Oleiros, Netbiblo.

IMSERSO (2013): Información estadística del Sistema para la Autonomía y Atención a la Dependencia. Situación a 31 de octubre de 2013, Imserso [rhttp://www.dependencia.imserso.es/ InterPresent1/groups/imserso/documents/ binario/im_079778.pdf〉].

MINISTERIO DE EMPLEO Y SEGURIDAD SOCIAL (2013): Servicio del hogar familiar 2013, [folleto], Ministerio de Empleo y Seguridad Social [‘http://www.empleo.gob.es/es/portada/ serviciohogar/serviciohogar.pdf`].

RODRÍGUEZ-PICAVEA, A. (2007): "Reflexiones en torno a la figura del asistente personal para la vida independiente y la promoción de la autonomía de las personas con diversidad funcional (discapacidad)", Zerbitzuan, no 41, págs. 115127 [<http://www.zerbitzuan.net/documentos/ zerbitzuan/Reflexiones\%20en\%2otorno\%20 a\%20la\%2ofigura\%20del\%20asistente.pdf $)$.

SIADECO (2012): Estudio cualitativo sobre el impacto de las prestaciones económicas de dependencia en Gipuzkoa, Donostia-San Sebastián, Diputación Foral de Gipuzkoa.

ZUBIRI, I. (dir.) (2009): “El impacto económico del gasto en política social de la Diputación Foral de Gipuzkoa", en Ponencias del proceso de reflexión / Hausnarketa prozesuaren txostenak, Donostia-San Sebastián, Diputación Foral de Gipuzkoa, págs. 351-452. 